\title{
ERROR ESTIMATE FOR A FULLY DISCRETE SPECTRAL SCHEME FOR KORTEWEG-DE VRIES-KAWAHARA EQUATION.
}

\author{
U. KOLEY
}

\begin{abstract}
We are concerned with the convergence of spectral method for the numerical solution of the initial-boundary value problem associated to the Korteweg-de Vries-Kawahara equation (in short Kawahara equation), which is a transport equation perturbed by dispersive terms of 3rd and 5th order. This equation appears in several fluid dynamics problems. It describes the evolution of small but finite amplitude long waves in various problems in fluid dynamics. These equations are discretized in space by the standard FourierGalerkin spectral method and in time by the explicit leap-frog scheme. For the resulting fully discrete, conditionally stable scheme we prove an $L^{2}$-error bound of spectral accuracy in space and of second-order accuracy in time.
\end{abstract}

\section{INTRODUCTION}

In this paper, we analyze the numerical approximation by Fourier spectral methods to the Korteweg-de Vries-Kawahara (briefly Kawahara) equation with periodic solutions:

$$
\begin{cases}u_{t}=-u u_{x}-u_{x x x}+u_{x x x x x}, & (x, t) \in \mathbb{R} \times[0, \infty), \\ u(x, t)=u(x+2 \pi, t), & (x, t) \in \mathbb{R} \times[0, \infty), \\ u(x, 0)=f(x), & x \in \mathbb{R},\end{cases}
$$

where the initial condition $f$ is a given real valued $2 \pi$-periodic function.

It is well known that the one-dimensional waves of small but finite amplitude in dispersive systems (e.g., the magneto-acoustic waves in plasmas, the shallow water waves, the lattice waves and so on) can be described by the Korteweg-de Vries (KdV in short) equation, given by

$$
u_{t}=-u u_{x}-u_{x x x},
$$

which admits either compressive or rarefactive steady solitary wave solution (by a solitary water wave, we mean a travelling wave solution of the water wave equations for which the free surface approaches a constant height as $|x| \rightarrow \infty$ ) according to the sign of the dispersion term (the third order derivative term). Under certain circumstances, however, it might happen that the coefficient of the third order derivative in the $\mathrm{KdV}$ equation becomes small or even zero. In that case one has to take account of the higher order effect of dispersion in order to balance the nonlinear effect. In such cases one may obtain a generalized nonlinear dispersive equation, known as Kawahara equation, which has a form of the KdV equation with an additional fifth order derivative term given by (1.1). The Kawahara equation is

Date: November 1, 2018.

Key words and phrases. Kawahara equation, Fourier-Galerkin spectral method, Error estimate, Convergence. 
an important nonlinear dispersive equation. It describes solitary wave propagation in media in which the first-order dispersion is anomalously small. A more specific physical background of this equation was introduced by Hunter and Scheurle [8], where they used it to describe the evolution of solitary waves in fluids in which the Bond number is less than but close to $\frac{1}{3}$ and the Froude number is close to 1 . In the literature this equation is also referred to as the fifth order $\mathrm{KdV}$ equation or singularly perturbed $\mathrm{KdV}$ equation. The fifth order term $\partial_{x}^{5} u$ is called the Kawahara term. There has been a great deal of work on solitary wave solutions of the Kawahara equation [11, 12, 9, 16, 6] over the past thirty years. It is found that, similarly to the KdV equation, the Kawahara equation also has solitary wave solutions which decay rapidly to zero as $t \rightarrow \infty$, but unlike the $\mathrm{KdV}$ equation whose solitary wave solutions are non-oscillating, the solitary wave solutions of the Kawahara equation have oscillatory trails. This shows that the Kawahara equation is not only similar but also different from the $\mathrm{KdV}$ equation in the properties of solutions, like what happens between the formulations of this equation and the $\mathrm{KdV}$ equation. The strong physical background of the Kawahara equation and such similarities and differences between it and the KdV equation in both the form and the behavior of the solution render the mathematical treatment of this equation particularly interesting. The Cauchy problem for Kawahara equation has been studied by a few authors $[7,20,10,21,4]$. It has been shown that the Cauchy problem has a local solution $u \in C\left([-T, T] ; H^{r}(\mathbb{R})\right)$ if $f \in H^{r}(\mathbb{R})$ and $r>-1$. This local result combined with the energy conservation law yields that (1.1) has a global solution $u \in C\left([-\infty, \infty] ; L^{2}(\mathbb{R})\right)$ if $f \in L^{2}(\mathbb{R})$. Well-posedness results can be found in [7].

Being integrable, Kawahara equation (1.1) has infinitely many invariants. Below we will state only first three of them.

Lemma 1.1. There exists a unique solution to (1.1). Moreover this solution conserves the first three energy integrals, namely

$$
\begin{aligned}
& (\partial / \partial t)\left[\int_{0}^{2 \pi} u(x, t) d x\right]=0, \\
& (\partial / \partial t)\left[\int_{0}^{2 \pi} u^{2}(x, t) d x\right]=0, \\
& (\partial / \partial t)\left[\int_{0}^{2 \pi}\left(\frac{1}{3} u^{3}-u_{x}^{2}-u_{x x}^{2}\right) d x\right]=0 .
\end{aligned}
$$

Proof. The invariance of these expressions can be shown for smooth solutions by using periodicity. For the sake of completeness, we will give a proof.

In order to show (1.3), let us integrate (1.1) in space. We get

$$
(\partial / \partial t) \int_{0}^{2 \pi} u d x+(1 / 2) \int_{0}^{2 \pi}\left(u^{2}\right)_{x} d x+\int_{0}^{2 \pi} u_{x x x} d x-\int_{0}^{2 \pi} u_{x x x x x} d x=0,
$$

using the periodicity of $u$, we deduce then (1.3).

To prove (1.4) we start by multyplying the equation (1.1) by $u$ and integrate by parts in space, yields

$$
\int_{0}^{2 \pi} u u_{t} d x=\int_{0}^{2 \pi}-u^{2} u_{x}-u u_{x x x}+u u_{x x x x x} d x
$$




$$
\begin{aligned}
= & -\int_{0}^{2 \pi}\left(\frac{1}{3} u^{3}\right)_{x} d x-\int_{0}^{2 \pi}\left(u u_{x x}-\frac{1}{2} u_{x}^{2}\right)_{x} d x-\int_{0}^{2 \pi} u_{x} u_{x x x x} d x \\
=- & \int_{0}^{2 \pi}\left(\frac{1}{3} u^{3}\right)_{x} d x-\int_{0}^{2 \pi}\left(u u_{x x}-\frac{1}{2} u_{x}^{2}\right)_{x} d x \\
& -\int_{0}^{2 \pi}\left(u_{x} u_{x x x}-\frac{1}{2} u_{x x}^{2}\right)_{x} d x .
\end{aligned}
$$

Again using the periodicity of $u$, we can establish (1.4).

To prove (1.5), we start by multyplying (1.1) by $u^{2}$ and integrate by parts in space, yields

$$
\begin{aligned}
\int_{0}^{2 \pi} u^{2} u_{t} d x & =\int_{0}^{2 \pi}-u^{3} u_{x}-u^{2} u_{x x x}+u^{2} u_{x x x x x} d x \\
& =-\int_{0}^{2 \pi}\left(\frac{1}{4} u^{4}\right)_{x} d x+2 \int_{0}^{2 \pi}\left(u u_{x}\right) u_{x x} d x-2 \int_{0}^{2 \pi}\left(u u_{x}\right) u_{x x x x} d x \\
& =2 \int_{0}^{2 \pi}\left[-u_{t}-u_{x x x}+u_{x x x x x}\right] u_{x x} d x \\
& -2 \int_{0}^{2 \pi}\left[-u_{t}-u_{x x x}+u_{x x x x x}\right] u_{x x x x} d x \\
& =2 \int_{0}^{2 \pi} u_{t x} u_{x} d x-2 \int_{0}^{2 \pi} u_{x x} u_{x x x} d x+2 \int_{0}^{2 \pi} u_{x x} u_{x x x x x x} d x \\
-2 & \int_{0}^{2 \pi} u_{t x} u_{x x x} d x+2 \int_{0}^{2 \pi} u_{x x x} u_{x x x x} d x-2 \int_{0}^{2 \pi} u_{x x x x} u_{x x x x x} d x \\
& =2 \int_{0}^{2 \pi} u_{t x} u_{x} d x+2 \int_{0}^{2 \pi} u_{t x x} u_{x x} d x .
\end{aligned}
$$

From this we can conclude that

$$
\partial / \partial t\left[\int_{0}^{2 \pi}\left(\frac{1}{3} u^{3}-u_{x}^{2}-u_{x x}^{2}\right) d x\right]=0 .
$$

There has been a great deal of work on the Fourier-Galerkin spectral method for the $\mathrm{KdV}$ equations $[1,2,14]$. Also, spectral methods for initial- and periodic boundary value problems for nonlinear wave equations with nonlocal dispersive terms has been studied by many authors [15]. In this paper, we prove error estimates for a simple spectral fully discrete scheme that we use to approximate spatially periodic solutions of Kawahara equation. We first discretize in space using the standard Fourier-Galerkin spectral method, which is easily shown to preserve the first three invariants of the equation. We prove that the solution of the semi discrete problems converges in $L^{2}$, on bounded temporal intervals, to the solution of the corresponding continuous problem at the spectral rate $N^{-r}$; here the number of Fourier points is $2 N+1$ and $r$ is the order of the Sobolev space in which the solution is supposed to belong for $t=0$.

We then discretize in time the ODE system that results from the spectral semidiscretization using two different methods. First, a second order accurate explicit scheme, the leap frog method and secondly, a second order accurate semi-implicit scheme, the Crank-Nicholson method. We prove the expected $\mathcal{O}\left(\Delta t^{2}\right)$ error bound 
in $L^{2}$ for both these temporal discretization, for suitably accurate initial conditions and under the stability requirement that $\Delta t N^{5}$ and $\Delta t N$ respectively are sufficiently small. The same type of mesh restriction is required for stability of any explicit temporal discretization of the stiff ODE semi discrete system under consideration.

The rest of the paper is organized as follows: In section 2, we give all the necessary preliminary results. In section 3, we consider a semi discrete FourierGalerkin scheme for the initial-boundary value problem corresponding to (1.1) and prove an error estimate. We prove that the solution of the semi discrete problem converges in $L^{2}$. In section 4 , we consider a fully discrete explicit Fourier-Galerkin scheme for the initial-boundary value problem corresponding to (1.1) and prove an error estimate under the stability condition that $\Delta t N^{5}$ is sufficiently small. Finally, in section 5, we consider a fully discrete semi explicit Fourier-Galerkin scheme for the initial-boundary value problem corresponding to (1.1) and prove an error estimate under the stability condition that $\Delta t N$ is sufficiently small.

\section{Notation and Preliminary Results}

We consider functions that are periodic of period $2 \pi$. The function spaces we use here are $L^{2}$ and the Sobolev spaces $H^{r}$ for integer $r \geq 0$. These spaces will always be considered on $[-\pi, \pi]$ and their elements will be periodic functions. We denote by $(.,$.$) the standard L^{2}$ inner product; this yields a norm in $L^{2}$ which we denote by $\|$.$\| . The norm in H^{r}$, denoted by $\|\cdot\|_{r}$, is defined by

$$
\|f\|_{r}=\left(\sum_{k \in \mathbb{Z}}\left(1+k^{2}\right)^{r}|\hat{f}(k)|^{2}\right)^{1 / 2} .
$$

By $\|\cdot\|_{\infty}$, we denote the norm of $L^{\infty}=L^{\infty}[-\pi, \pi]$.

As usual, we denote by $\hat{f}(k), k \in \mathbb{Z}$, the Fourier coefficients of $f$ :

$$
\hat{f}(k)=\frac{1}{2 \pi} \int_{-\pi}^{\pi} e^{-i k x} f(x) d x .
$$

We recall that the Fourier coefficients of the pointwise product are given by the convolution of the Fourier coefficients of $f$ and $g$, defined by

$$
(\hat{f} * \hat{g})(k)=\sum_{m, n \in \mathbb{Z} ; m+n=k} \hat{f}(m) \hat{g}(n) .
$$

We also need to consider discrete analogues of the quantities defined above. To this end, for $N$ a positive integer, consider the space $S_{N}$ defined by

$$
S_{N}=\operatorname{span}\{\exp (i k x): k \in \mathbb{Z},-N \leq k \leq N\} .
$$

Let $P_{N}$ denote the $L^{2}$ orthogonal projection onto $S_{N}$. The projection has the following approximation properties, whose proof is standard.

Proposition 2.1. Given integers $0 \leq s \leq r$, there exists a constant $C$ independent of $N$ such that, for any $f \in H^{r}$,

$$
\begin{aligned}
& \left\|f-P_{N} f\right\|_{s} \leq C N^{s-r}\|f\|_{r} \\
& \left\|f-P_{N} f\right\|_{\infty} \leq C N^{1 / 2-r}\|f\|_{r}, \quad r \geq 1 .
\end{aligned}
$$


Proposition 2.2. Given integers $0 \leq s \leq r$, there exists a constant $C$ independent of $N$ such that, for any $\psi \in S_{N}$,

$$
\|\psi\|_{r} \leq C N^{r-s}\|\psi\|_{s}, \quad \text { and } \quad\|\psi\|_{\infty} \leq C N^{1 / 2}\|\psi\| .
$$

We use many times in the proofs a Sobolev-type inequality, which states that there exists a constant $C>0$ such that for all $f \in H^{1}$

$$
\|f\|_{\infty} \leq C\|f\|^{1 / 2}\|f\|_{1}^{1 / 2} \text {. }
$$

As we have already mentioned in the introduction, we shall consider the initialand periodic boundary-value problem for the Kawahara equation: We seek a realvalued function $u(x, t), 2 \pi$ periodic in $x$ and satisfying

$$
\begin{cases}u_{t}+u u_{x}+u_{x x x}=u_{x x x x x}, & x \in[-\pi, \pi], \quad t \geq 0, \\ u(x, 0)=f(x), & x \in[-\pi, \pi] .\end{cases}
$$

Here $f(x)$ is a real-valued, $2 \pi$ periodic function.

We now going to state a existence and uniqueness result for the solution of (2.7). See [13] for a proof.

Theorem 2.1. Let $f$ be in $H^{r}$, with $r \geq 5$. Then there exists a unique solution $u$ of $(2.7)$ in $H^{r}$, which belongs to the space $C^{k}\left([0, \infty) ; H^{r-5 k}\right)$ with $r-5 k \geq-1$, i.e., is such that its temporal derivatives up to order $k$ exist and are continuous and bounded on $[0, \infty)$ with values in $H^{r-5 k}$.

\section{Semi-Discrete Approximation:}

In this section we analyze a Fourier-Galerkin scheme for the discretization of (1.1) in the spatial variable.

The semi discrete Fourier-Galerkin (spectral) approximation to (1.1) is a map U from $[0, \infty)$ to the real-valued elements of $S_{N}$ such that, for all $\phi \in S_{N}$ :

$$
\left\{\begin{array}{l}
\left(\mathbf{U}_{t}+\mathbf{U U}_{x}+\mathbf{U}_{x x x}-\mathbf{U}_{x x x x x}, \phi\right)=0, \quad t \geq 0, \\
\mathbf{U}(0)=P_{N} f,
\end{array}\right.
$$

where $P_{N}$ is the orthogonal projection of $L^{2}$ onto $S_{N}$.

By choosing $\phi=e^{i k x}$ for $k=-N, \cdots, N$, we see that (3.1) is an initial-value problem for an ODE system for Fourier coefficients $\hat{\mathbf{U}}(k, t)$ of $\mathbf{U}$. Since $\mathbf{U}$ is real, these coefficients must satisfy the condition $\hat{\mathbf{U}}(k, t)=\overline{\hat{\mathbf{U}}(k, t)}$ and the equation

$$
\begin{aligned}
\hat{\mathbf{U}}_{t}(k, t) & =\frac{-i k}{2} \hat{\mathbf{U}} * \hat{\mathbf{U}}(k, t)-k^{3} \hat{\mathbf{U}}(k, t)-k^{5} \hat{\mathbf{U}}(k, t), \quad-N \leq k \leq N \\
\hat{\mathbf{U}}(k, 0) & =\hat{f}(k) .
\end{aligned}
$$

The right hand side of the system (3.2) is Lipschitz continuous, at least locally, with respect to $l^{2}$ norm. Hence, the existence of a maximal time $t_{0}, 0<t_{0} \leq T$ such that, for all $t<t_{0}$, there exists a unique solution $U(t)$ to problem (3.1) is a classical result of the theory of differential systems. The problem is to get the existence for an arbitrary time $t_{0}$, or equivalently to prove that one can take $t_{0}=T$. This result is a consequence of the fact that (3.1) is conservative in $L^{2}$, which ensures that the solution cannot blow-up.

Now we will present the main properties enjoyed by the Fourier-Galerkin approximation (3.1). More precisely, this semidiscretization preserves the discrete analogues of the first three invariants of (2.7). 
Lemma 3.1. There exists a unique solution $\mathbf{U}$ to problem (3.1). Moreover this solution conserves the first three energy integrals of Kawahara equation, namely

$$
\begin{aligned}
& (\partial / \partial t)\left[\int_{-\pi}^{\pi} \mathbf{U}(x, t) d x\right]=0 \\
& (\partial / \partial t)\left[\int_{-\pi}^{\pi} \mathbf{U}^{2}(x, t) d x\right]=0 \\
& (\partial / \partial t)\left[\int_{-\pi}^{\pi}\left(\frac{1}{3} \mathbf{U}^{3}-\mathbf{U}_{x}^{2}-\mathbf{U}_{x x}^{2}\right)(x, t) d x\right]=0 .
\end{aligned}
$$

Proof. First of all, we have already discussed about the existence of a unique solution to (3.1). Now in order to show (3.3), let us first choose $\phi=1$ as a test function in (3.1). We get

$$
\begin{aligned}
(\partial / \partial t) \int_{-\pi}^{\pi} \mathbf{U}(x, t) d x+(1 / 2) \int_{-\pi}^{\pi} \mathbf{U}_{x}^{2}(x, t) d x & +\int_{-\pi}^{\pi} \mathbf{U}_{x x x}(x, t) d x \\
& -\int_{-\pi}^{\pi} \mathbf{U}_{x x x x x}(x, t) d x=0,
\end{aligned}
$$

using the periodicity of $\mathbf{U}$, we deduce then (3.3). To prove (3.4), we choose $\phi=\mathbf{U}$ in (3.1). We obtain

$$
\begin{aligned}
(\partial / \partial t) \int_{-\pi}^{\pi} \mathbf{U}^{2}(x, t) d x+(1 / 3) \int_{-\pi}^{\pi} \mathbf{U}_{x}^{3}(x, t) d x & +\int_{-\pi}^{\pi}\left(\mathbf{U} \mathbf{U}_{x x x}\right)(x, t) d x \\
& -\int_{-\pi}^{\pi}\left(\mathbf{U} \mathbf{U}_{x x x x x}\right)(x, t) d x=0 .
\end{aligned}
$$

Integrating by parts and using the periodicity of $\mathbf{U}$ yields

$$
\int_{-\pi}^{\pi}\left(\mathbf{U U}_{x x x}\right)(x, t) d x=-(1 / 2) \int_{-\pi}^{\pi}\left(\mathbf{U}_{x}^{2}\right)_{x}(x, t) d x=0 .
$$

Similarly, we have

$$
\int_{-\pi}^{\pi}\left(\mathbf{U U}_{x x x x x}\right)(x, t) d x=0, \quad \text { and } \quad \int_{-\pi}^{\pi} \mathbf{U}_{x}^{3}(x, t) d x=0 .
$$

Hence, we deduce (3.4).

We derive now (3.5) by choosing $\phi=P_{N}\left(\mathbf{U}^{2}\right)$ in (3.1). As a result we obtain

$$
\begin{aligned}
\int_{-\pi}^{\pi} \mathbf{U}_{t} P_{N} \mathbf{U}^{2} d x+\int_{-\pi}^{\pi} \mathbf{U} \mathbf{U}_{x} P_{N} \mathbf{U}^{2} d x & +\int_{-\pi}^{\pi} \mathbf{U}_{x x x} P_{N} \mathbf{U}^{2} d x \\
& -\int_{-\pi}^{\pi} \mathbf{U}_{x x x x x} P_{N} \mathbf{U}^{2} d x=0 .
\end{aligned}
$$

Now as $\mathbf{U}_{t}$ is an element of $S_{N}$, we have

$$
\int_{-\pi}^{\pi} \mathbf{U}_{t} P_{N} \mathbf{U}^{2} d x=\int_{-\pi}^{\pi} \mathbf{U}_{t} \mathbf{U}^{2} d x=(1 / 3)(\partial / \partial t) \int_{-\pi}^{\pi} \mathbf{U}^{3} d x .
$$

On the other hand,

$$
\int_{-\pi}^{\pi} \mathbf{U}_{x x x} P_{N} \mathbf{U}^{2} d x=\int_{-\pi}^{\pi} \mathbf{U}_{x x x} \mathbf{U}^{2} d x=-2 \int_{-\pi}^{\pi} \mathbf{U}_{x x}\left(\mathbf{U} \mathbf{U}_{x}\right) d x
$$




$$
=-\int_{-\pi}^{\pi} \mathbf{U}_{x x}\left(-\mathbf{U}_{t}-\mathbf{U}_{x x x}+\mathbf{U}_{x x x x x}\right) d x=-(\partial / \partial t) \int_{-\pi}^{\pi} \mathbf{U}_{x}^{2} d x .
$$

Similarly we have

$$
-\int_{-\pi}^{\pi} \mathbf{U}_{x x x x x} P_{N} \mathbf{U}^{2} d x=-(\partial / \partial t) \int_{-\pi}^{\pi} \mathbf{U}_{x x}^{2} d x .
$$

Finally, using the fact that $P_{N}$ commutes with differentiation, we obtain

$$
\begin{aligned}
& \int_{-\pi}^{\pi} \mathbf{U} \mathbf{U}_{x} P_{N} \mathbf{U}^{2} d x=\int_{-\pi}^{\pi}\left(\frac{1}{2} \mathbf{U}^{2}\right)_{x} P_{N} \mathbf{U}^{2} d x \\
& =-\int_{-\pi}^{\pi} \mathbf{U}^{2} P_{N}\left(\mathbf{U} \mathbf{U}_{x}\right) d x=-\int_{-\pi}^{\pi} \mathbf{U} \mathbf{U}_{x} P_{N} \mathbf{U}^{2} d x,
\end{aligned}
$$

consequently, we have

$$
\int_{-\pi}^{\pi} \mathbf{U} \mathbf{U}_{x} P_{N} \mathbf{U}^{2} d x=0
$$

Combining all the results above we get (3.5).

We shall now state the following theorem:

Theorem 3.1. The semi-discrete scheme (3.1) has an unique solution $\mathbf{U}$ for $t \geq 0$. Let $u(x, t)$ be the solution of (1.1) corresponding to the initial data $u_{0} \in H^{r}$. Then there exists a time $T>0$, and a constant $C>0$, independent of $N$, such that

$$
\max _{0 \leq t \leq T}\|u-\mathbf{U}\| \leq \frac{C}{N^{r}} .
$$

Before giving the proof of Theorem 3.1, we define a different semi-discretization based on a linearization of (3.1). The convergence of this approximation will serve as an intermediate step in the proof of the convergence of the original scheme.

To this end, we linearize (3.1) as follows: Given a solution of (1.1), corresponding to initial data $u_{0}$ in $H^{r}$, we look for a function $V \in S_{N}$, which for all $\phi \in S_{N}$, satisfies

$$
\left\{\begin{array}{l}
\left(V_{t}+u V_{x}+V_{x x x}-V_{x x x x x}, \phi\right)=0, \quad t \geq 0, \\
V(0)=P_{N} u_{0},
\end{array}\right.
$$

Lemma 3.2. Let $u(x, t)$ be a solution of (1.1) corresponding to initial data $u_{0} \in$ $H^{r}$. Then there exists a unique solution $V$ of (3.7) for all $t \geq 0$. Moreover, given $0 \leq t \leq T$, there exists a constant $C$ independent of $N$ such that

$$
\max _{0 \leq t \leq T}\|u-V\| \leq \frac{C}{N^{r}}
$$

Proof. The existence of an unique local solution of (3.7) is again a consequence of standard ODE theory. To see that we have global existence, we resort to a stability result in the $L^{2}$ norm. Choosing $\phi=V$ in (3.7), we obtain

$$
\frac{1}{2} \frac{d}{d t}\|V\|^{2} \leq\left\|u_{x}\right\|_{\infty}\|V\|^{2} .
$$

Sobolev's inequality and the fact that $u_{0} \in H^{2}$ imply that $\max _{t \geq 0}\left\|u_{x}\right\|_{\infty} \leq C$; thus by the Gronwall inequality, there exists $C$ such that $\max _{0 \leq t \leq T}\|V\| \leq C$, and we can extend the local solution to a solution on every bounded interval $[0, T]$. 
Note that using the same arguments and choosing $\phi=V_{x x}$, we conclude that $\max _{0 \leq t \leq T}\left\|V_{x}\right\| \leq C$.

Now set $\rho=P_{N} u-V$, then $u-V=u-P_{N} u+P_{N} u-V=u-P_{N} u+\rho$. The hypothesis on $u$ imply that $\left\|u-P_{N} u\right\| \leq C / N^{r}$. Thus we only need to estimate $\rho$. Observe that, $\rho$ is an element of $S_{N}$ satisfying the equation

$$
\left(\rho_{t}+\rho_{x x x}-\rho_{x x x x x}, \phi\right)=-\left(P_{N}\left(u u_{x}\right), \phi\right)+\left(\rho u V_{x}, \phi\right) .
$$

Now observe that,

$$
u u_{x}-u V_{x}=\left(u-P_{N} u\right) u_{x}+\left(P_{N} u-V\right) u_{x}+V u_{x}-u V_{x}
$$

Then, the choice $\phi=\rho$ yields,

$\frac{1}{2} \frac{d}{d t}\|\rho\|^{2} \leq\left\|u_{x}\right\|_{\infty}\left\|u-P_{N} u\right\|\|\rho\|+\left\|u_{x}\right\|_{\infty}\|\rho\|^{2}+\left\|u_{x}\right\|_{\infty}\|\rho\|\|V\|+\|u\|_{\infty}\|\rho\|\left\|V_{x}\right\|$, so that by the arithmetic-geometric inequality we obtain,

$$
\frac{1}{2} \frac{d}{d t}\|\rho\|^{2} \leq \frac{C}{\left(N^{r}\right)^{2}}+C\|\rho\|^{2} .
$$

But since $\rho(x, 0)=0$, hence we obtain by using Grownwall's inequality

$$
\max _{0 \leq t \leq T}\|\rho\| \leq \frac{C}{N^{r}}
$$

and this completes the proof of the lemma.

Proof. (of Theorem 3.1)

We have already proved the existence and uniqueness of the semi-discrete solution

$\mathbf{U}$. Now set $e=V-\mathbf{U}$. Then $u-\mathbf{U}=u-V+e$. In view of (3.8), we only need an estimate for $e$. Now observe that $e$ satisfies

$$
\left(e_{t}+e_{x x x}-e_{x x x x x}, \phi\right)=-\left(u V_{x}-\mathbf{U U}_{x}, \phi\right) .
$$

Now using the fact that

$$
u V_{x}-\mathbf{U U}_{x}=(u-V) V_{x}+(e V)_{x}-e e_{x}
$$

and choosing $\phi=e$, observing that $\left(e, e e_{x}\right)=0$, yields,

$$
\frac{1}{2} \frac{d}{d t}\|e\|^{2} \leq\left\|V_{x}\right\|_{\infty}\|u-V\|\|e\|+\frac{1}{2}\left\|V_{x}\right\|_{\infty}\|e\|^{2} .
$$

We now use the following inverse inequalities: for $0 \leq s \leq r$ and $\psi \in S_{N}$

$$
\|\psi\|_{r} \leq C N^{r-s}\|\psi\|_{s}, \quad\|\psi\|_{\infty} \leq C N^{1 / 2}\|\psi\| .
$$

Since $\left(P_{N} u-V\right)$ is in $S_{N}$,

$$
\left\|\left(P_{N} u-V\right)_{x}\right\|_{\infty} \leq C\left\|P_{N} u-V\right\|_{1}^{1 / 2}\left\|P_{N} u-V\right\|_{2}^{1 / 2} \leq C N^{3 / 2}\left\|P_{N} u-V\right\| .
$$

Consequently, we have

$$
\begin{aligned}
&\left\|P_{N} u-V\right\|_{\infty} \leq C N^{1 / 2-r}, \\
&\left\|\left(P_{N} u-V\right)_{x}\right\|_{\infty} \leq C N^{3 / 2-r} .
\end{aligned}
$$

Using Sobolev's inequality along with the approximation properties of the projection $P_{N}$, we have

$$
\begin{aligned}
\left\|V_{x}\right\|_{\infty} & \leq\left\|u_{x}\right\|_{\infty}+\left\|\left(u-P_{N} u\right)_{x}\right\|_{\infty}+\left\|\left(P_{N} u-V\right)_{x}\right\|_{\infty} \\
& \leq C+C\left\|u-P_{N} u\right\|_{2}+\frac{C}{N^{r-3 / 2}}
\end{aligned}
$$




$$
\leq C+\frac{C}{N^{r-2}}+\frac{C}{N^{r-3 / 2}} .
$$

Using $r \geq 2$, these inequalities yield

$$
\max _{0 \leq t \leq T}\left\|V_{x}\right\|_{\infty} \leq C,
$$

consequently (using arithmetic-geometric inequality), from (3.9)

$$
\frac{1}{2} \frac{d}{d t}\|e\|^{2} \leq C\left(\|e\|^{2}+\|u-V\|^{2}\right) .
$$

Finally, writing $u-V=u-P_{N} u+\rho$, we have

$$
\frac{1}{2} \frac{d}{d t}\|e\|^{2} \leq C\|e\|^{2}+\left(\frac{C}{N^{r}}\right)^{2} .
$$

Since $e(0)=0$, Gronwall's inequality gives,

$$
\max _{0 \leq t \leq T}\|e\| \leq \frac{C}{N^{r}},
$$

which in view of (3.8), yields (3.6).

\section{Fully-Discrete scheme:}

To define the fully discrete scheme, given $0<T<\infty$, choose a time step $\Delta t$, and an integer $M$, such that $M \Delta t=T$. Then for $m=0, \cdots, M$, denote $t_{m}=m \Delta t$. The fully discrete solution is defined as the sequence $\left\{\mathbf{U}^{m}\right\}$ of elements of $S_{N}$ satisfying, for all $\phi \in S_{N}$ and for $m=1,2, \cdots, M$, the equation

$$
\left(\mathbf{U}^{m+1}-\mathbf{U}^{m-1}, \phi\right)+2 \Delta t\left(\mathbf{U}^{m} \mathbf{U}_{x}^{m}+\mathbf{U}_{x x x}^{m}-\mathbf{U}_{x x x x x}^{m}, \phi\right)=0 .
$$

For each $m, \mathbf{U}^{m}$ is an approximation of $\mathbf{U}\left(t_{m}\right)$, the semi-discrete solution $\mathbf{U}$ evaluated at time $t=t_{m}$. We also suppose that initial values $\mathbf{U}^{0}, \mathbf{U}^{1}$ have been given in $S_{N}$.

Theorem 4.1. Let $\mathbf{U}(t)$ be the solution of the semi-discrete problem (3.1) and $\left\{\mathbf{U}^{m}\right\}$ be the solution of (4.1). Suppose that $\mathbf{U}^{0}=\mathbf{U}(0)$ and that $\mathbf{U}^{1}$ is computed in such a way that

$$
\left\|\mathbf{U}^{1}-\mathbf{U}(\Delta t)\right\| \leq C \Delta t^{2}
$$

Assume that $u_{0}$ is in $H^{r}$ with $r \geq 16$. Then, there exists a constant $C_{1}$ independent of $N$ and $\Delta t$, such that if

$$
N^{5} \Delta t \leq C_{1}
$$

there holds

$$
\max _{0 \leq m \leq M}\left\|\mathbf{U}^{m}-\mathbf{U}\left(t_{m}\right)\right\| \leq C \Delta t^{2}
$$

Proof. We see that $\left\{\mathbf{U}^{m}\right\}$ satisfies for all $\phi \in S_{N}$ :

$$
\left(\mathbf{U}^{m+1}-\mathbf{U}^{m-1}, \phi\right)+2 \Delta t\left(\mathbf{U}^{m} \mathbf{U}_{x}^{m}+\mathbf{U}_{x x x}^{m}-\mathbf{U}_{x x x x x}^{m}, \phi\right)=0 .
$$

On the other hand, the semi-discrete solution $\mathbf{U}$ satisfies, for all $\phi \in S_{N}$ :

$$
\begin{aligned}
\left(\mathbf{U}\left(t_{m+1}\right)-\mathbf{U}\left(t_{m-1}\right), \phi\right)+2 \Delta t\left(\mathbf{U}\left(t_{m}\right) \mathbf{U}_{x}\left(t_{m}\right)+\mathbf{U}_{x x x}\left(t_{m}\right)-\right. & \left.\mathbf{U}_{x x x x x}\left(t_{m}\right), \phi\right) \\
& =\left(\theta^{m}, \phi\right),
\end{aligned}
$$


where $\theta^{m}$ is an element of $S_{N}$ given by:

$$
\theta^{m}=\mathbf{U}\left(t_{m+1}\right)-\mathbf{U}\left(t_{m-1}\right)-2 \Delta t \mathbf{U}_{t}\left(t_{m}\right) .
$$

From Taylor's expansion, we have

$$
\left\|\theta^{m}\right\| \leq C \Delta t^{3} \max _{t_{m-1} \leq s \leq t_{m+1}}\left\|\frac{\partial^{3} \mathbf{U}(s)}{\partial t^{3}}\right\| .
$$

Now let us define $e^{m} \in S_{N}$ as

$$
e^{m}=\mathbf{U}^{m}-\mathbf{U}\left(t_{m}\right) .
$$

Then $e^{m}$ satisfies, for all $\phi \in S_{N}$

$$
\left(e^{m+1}-e^{m-1}, \phi\right)+2 \Delta t\left(\mathbf{U}^{m} \mathbf{U}_{x}^{m}-\mathbf{U}\left(t_{m}\right) \mathbf{U}_{x}\left(t_{m}\right)+e_{x x x}^{m}-e_{x x x x x}^{m}, \phi\right)=-\left(\theta^{m}, \phi\right) .
$$

Choosing $\phi=e^{m+1}+e^{m-1}$, and adding $\left\|e^{m}\right\|^{2}$ to both sides of (4.8), we obtain

$$
\begin{aligned}
& \left\|e^{m+1}\right\|^{2}+\left\|e^{m}\right\|^{2}-2 \Delta t\left(e_{x x}^{m}, e_{x}^{m+1}\right)-2 \Delta t\left(e_{x x x}^{m}, e_{x x}^{m+1}\right) \\
& =\left\|e^{m}\right\|^{2}+\left\|e^{m-1}\right\|^{2}-2 \Delta t\left(e_{x x}^{m-1}, e_{x}^{m}\right)-2 \Delta t\left(e_{x x x}^{m-1}, e_{x x}^{m}\right)-\left(\theta^{m}, e^{m+1}+e^{m-1}\right) \\
& -2 \Delta t\left(\mathbf{U}_{x}^{m} e^{m}, e^{m+1}+e^{m-1}\right)-2 \Delta t\left(\mathbf{U}\left(t_{m}\right) e_{x}^{m}, e^{m+1}+e^{m-1}\right),
\end{aligned}
$$

where we have used the following identity

$$
\mathbf{U}^{m} \mathbf{U}_{x}^{m}-\mathbf{U}\left(t_{m}\right) \mathbf{U}_{x}\left(t_{m}\right)=\mathbf{U}_{x}^{m} e^{m}+\mathbf{U}\left(t_{m}\right) e_{x}^{m} .
$$

Now let us define $A^{m+1}$ by

$$
\begin{aligned}
A^{m+1}=\left\|e^{m+1}\right\|^{2}+\left\|e^{m}\right\|^{2} & -2 \Delta t\left(e_{x x}^{m}, e_{x}^{m+1}\right)-2 \Delta t\left(e_{x x x}^{m}, e_{x x}^{m+1}\right) \\
& -2 \Delta t\left(\mathbf{U}\left(t_{m}\right) e_{x}^{m+1}, e^{m}\right) .
\end{aligned}
$$

Then we can rewrite (4.9) as

$$
\begin{aligned}
A^{m+1}=A^{m} & -2 \Delta t\left(\mathbf{U}_{x}^{m} e^{m}, e^{m+1}+e^{m-1}\right)+2 \Delta t\left(\mathbf{U}_{x}\left(t_{m}\right) e^{m}, e^{m+1}\right) \\
& -2 \Delta t\left(\left(\mathbf{U}\left(t_{m}\right)-\mathbf{U}\left(t_{m-1}\right)\right) e_{x}^{m}, e^{m-1}\right)-\left(\theta^{m}, e^{m+1}+e^{m-1}\right) .
\end{aligned}
$$

In general, differentiating (3.1) with respect to $t$ and using the properties of $P_{N}$, it is straightforward to prove that there exist constants $\alpha_{k, s}$, independent of $N$, such that, if $r \geq s+5 k+1$

$$
\max _{0 \leq t \leq T}\left\|\partial_{t}^{k} \mathbf{U}(t)\right\|_{s} \leq \alpha_{k, s}
$$

In particular, since we assume $r \geq 16$, we have by Sobolev's inequality

$$
\left\|\mathbf{U}\left(t_{m}\right)-\mathbf{U}\left(t_{m-1}\right)\right\| \leq \Delta t \max _{t_{m-1} \leq s \leq t_{m}}\left\|\mathbf{U}_{t}(s)\right\|_{\infty} \leq C \Delta t,
$$

and

$$
\left\|\theta^{m}\right\| \leq C \Delta t^{3} .
$$

Again, (4.12) gives after some manipulations:

$$
\begin{aligned}
A^{m+1} \leq A^{m} & \left.\left.+C \Delta t^{2} N\left\|e^{m}\right\| \| e^{m+1}+e^{m-1}\right)\|+C \Delta t\| \mathbf{U}_{x}^{m}\left\|_{\infty}\right\| e^{m}\|\| e^{m+1}+e^{m-1}\right) \| \\
& \left.+C \Delta t\left\|e^{m}\right\|\left\|e^{m+1}\right\|+C \Delta t^{3} \| e^{m+1}+e^{m-1}\right) \| .
\end{aligned}
$$


Now as an internl "inductive" hypothesis, we assume that there exists a constant $B$, independent of $N$, such that for all $n \leq m$

$$
\left\|\mathbf{U}_{x}^{m}\right\|_{\infty} \leq B
$$

then using the Cauchy-Schwartz inequality, we have

$$
A^{m+1} \leq A^{m}+C \Delta t^{5}+C \Delta t(1+B+N \Delta t)\left(\left\|e^{m-1}\right\|^{2}+2\left\|e^{m}\right\|^{2}+\left\|e^{m+1}\right\|^{2}\right) .
$$

On the other hand, one can show that under a stability assumption (4.3), $A^{m+1}$ is positive and comparable to $\left\|e^{m}\right\|^{2}+\left\|e^{m+1}\right\|^{2}$. Infact,

$$
\begin{aligned}
\Delta t \mid\left(e_{x x}^{m}, e_{x}^{m+1}\right)+\left(e_{x x x}^{m}, e_{x x}^{m+1}\right) & +\left(\mathbf{U}\left(t_{m}\right) e_{x}^{m+1}, e^{m}\right) \mid \\
& \leq C \Delta t\left(N^{3}+N^{5}\right)\left(\left\|e^{m}\right\|^{2}+\left\|e^{m+1}\right\|^{2}\right) .
\end{aligned}
$$

Hence, if

$$
C \Delta t\left(N^{3}+N^{5}\right) \leq \frac{1}{2}
$$

i.e., under a condition of the form (4.3), we have

$$
\frac{1}{2}\left(\left\|e^{m}\right\|^{2}+\left\|e^{m+1}\right\|^{2}\right) \leq A^{m+1} \leq 2\left(\left\|e^{m}\right\|^{2}+\left\|e^{m+1}\right\|^{2}\right) .
$$

Consequently by (4.14), we have

$$
A^{m+1} \leq A^{m}+C \Delta t^{5}+C \Delta t(C+B)\left(A^{m}+A^{m+1}\right),
$$

which implies, for $C^{*}=C(C+B)$

$$
\left(1-C^{*} \Delta t\right) A^{m+1} \leq\left(1+C^{*} \Delta t\right) A^{m}+C \Delta t^{5} .
$$

Hence, if $\Delta t$ is chosen small enough, and using the fact that $\left\|e^{1}\right\|=\mathcal{O}\left(\Delta t^{2}\right)$ by (4.2), we obtain in the standard way

$$
\max _{1 \leq n \leq m+1}\left\|e^{n}\right\| \leq C \Delta t^{2} e^{C T}
$$

Observe that, the above estimate allows us to complete the inductive step. Indeed, we see that

$$
\left\|\mathbf{U}_{x}^{m+1}\right\|_{\infty} \leq\left\|\mathbf{U}_{x}\left(t_{m+1}\right)\right\|_{\infty}+\left\|e_{x}^{m+1}\right\|_{\infty} \leq\left\|\mathbf{U}_{x}\left(t_{m+1}\right)\right\|_{\infty}+C \Delta t^{2} N^{3 / 2} e^{C T} \leq B
$$

consequently, by taking $\Delta t$ sufficiently small and using a condition of type (4.3), we can justify the assumption that $\left\|\mathbf{U}_{x}^{m}\right\|_{\infty} \leq B$ for all $m$.

Remark 4.1. In conclusion, combining the results of Theorems 3.1 and 4.1, we see that under the hypotheses of Theorem 4.1, the fully discrete scheme (4.1) satisfies the error estimate

$$
\max _{0 \leq m \leq M}\left\|u\left(t_{m}\right)-\mathbf{U}^{m}\right\| \leq C\left(\frac{1}{N^{r}}+\Delta t^{2}\right)
$$




\section{SEMI-IMPLiCIT SCHEME}

To define the semi-implicit fully discrete scheme, given $0<T<\infty$, choose a time step $\Delta t$, and an integer $M$, such that $M \Delta t=T$. Then for $m=0, \cdots, M$, denote $t_{m}=m \Delta t$. The fully discrete solution is defined as the sequence $\left\{\mathbf{U}^{m}\right\}$ of elements of $S_{N}$ satisfying, for all $\phi \in S_{N}$ and for $m=1,2, \cdots, M$, the equation

$$
\left(\mathbf{U}^{m+1}-\mathbf{U}^{m}, \phi\right)+\Delta t\left(\mathbf{U}^{m+1 / 2} \mathbf{U}_{x}^{m+1 / 2}+\mathbf{U}_{x x x}^{m+1 / 2}-\mathbf{U}_{x x x x}^{m+1 / 2}, \phi\right)=0 .
$$

For each $m, \mathbf{U}^{m}$ is an approximation of $\mathbf{U}\left(t_{m}\right)$, the semi-discrete solution $\mathbf{U}$ evaluated at time $t=t_{m}$. Also, we have used the notation: $\mathbf{U}^{m+1 / 2}=\frac{1}{2}\left(\mathbf{U}^{m}+\mathbf{U}^{m+1}\right)$.

First we shall establish the rate of convergence estimates. So, for the time being, we assume the existence of a sequence $\left\{\mathbf{U}^{n}\right\}_{n=0}^{M}$ in $S_{N}$ satisfying (5.1). Later in this section we will discuss about the existence and uniqueness of such sequences.

Theorem 5.1. Let $\mathbf{U}(t)$ be the solution of the semi-discrete problem (3.1) and $\left\{\mathbf{U}^{m}\right\}$ be the solution of (4.1). Assume that $u_{0}$ is in $H^{r}$ with $r \geq 16$. Then, there exists a constant $C_{1}$ independent of $N$ and $\Delta t$, such that if

$$
N \Delta t \leq C_{1},
$$

there holds

$$
\max _{0 \leq m \leq M}\left\|\mathbf{U}^{m}-\mathbf{U}\left(t_{m}\right)\right\| \leq C \Delta t^{2}
$$

Proof. First note that, by letting $\phi=\mathbf{U}^{m+1 / 2}$ in (5.1), we have by periodicity

$$
\begin{aligned}
\frac{1}{2}\left(\left\|\mathbf{U}^{m+1}\right\|^{2}-\left\|\mathbf{U}^{m}\right\|^{2}\right) & =-\left(\mathbf{U}^{m+1 / 2} \mathbf{U}_{x}^{m+1 / 2}+\mathbf{U}_{x x x}^{m+1 / 2}-\mathbf{U}_{x x x x x}^{m+1 / 2}, \mathbf{U}^{m+1 / 2}\right) \\
& =0,
\end{aligned}
$$

hence

$$
\left\|\mathbf{U}^{m}\right\|=\left\|\mathbf{U}^{0}\right\|, \quad m=0, \cdots, M \text {. }
$$

We see that $\left\{\mathbf{U}^{m}\right\}$ satisfies for all $\phi \in S_{N}$ :

$$
\left(\mathbf{U}^{m+1}-\mathbf{U}^{m}, \phi\right)+\Delta t\left(\mathbf{U}^{m+1 / 2} \mathbf{U}_{x}^{m+1 / 2}+\mathbf{U}_{x x x}^{m+1 / 2}-\mathbf{U}_{x x x x x}^{m+1 / 2}, \phi\right)=0 .
$$

On the other hand, the semi-discrete solution $\mathbf{U}$ satisfies, for all $\phi \in S_{N}$ :

$$
\begin{aligned}
& \left(\mathbf{U}\left(t_{m+1}\right)-\mathbf{U}\left(t_{m}\right), \phi\right) \\
& +\Delta t\left(\mathbf{U}\left(t_{m+1 / 2}\right) \mathbf{U}_{x}\left(t_{m+1 / 2}\right)+\mathbf{U}_{x x x}\left(t_{m+1 / 2}\right)-\mathbf{U}_{x x x x x}\left(t_{m+1 / 2}\right), \phi\right)=\left(\theta^{m}, \phi\right),
\end{aligned}
$$

where $\theta^{m}$ is an element of $S_{N}$ given by:

$$
\theta^{m}=\mathbf{U}\left(t_{m+1}\right)-\mathbf{U}\left(t_{m}\right)-2 \Delta t \mathbf{U}_{t}\left(t_{m+1 / 2}\right) .
$$

From Taylor's expansion, we have

$$
\left\|\theta^{m}\right\| \leq C \Delta t^{3} \max _{t_{m-1} \leq s \leq t_{m+1}}\left\|\frac{\partial^{3} \mathbf{U}(s)}{\partial t^{3}}\right\| .
$$

Now let us define $e^{m} \in S_{N}$ as

$$
e^{m}=\mathbf{U}^{m}-\mathbf{U}\left(t_{m}\right) .
$$


Then $e^{m}$ satisfies, for all $\phi \in S_{N}$

$$
\begin{aligned}
& \left(e^{m+1}-e^{m}, \phi\right) \\
& +\Delta t\left(\mathbf{U}^{m+1 / 2} \mathbf{U}_{x}^{m+1 / 2}-\mathbf{U}\left(t_{m+1 / 2}\right) \mathbf{U}_{x}\left(t_{m+1 / 2}\right)+e_{x x x}^{m+1 / 2}-e_{x x x x x}^{m+1 / 2}, \phi\right)=-\left(\theta^{m}, \phi\right) .
\end{aligned}
$$

Now choose $\phi=e^{m+1 / 2}$ in (5.7) and observe the following estimates:

$$
\left(e^{m+1}-e^{m}, e^{m+1 / 2}\right)=\frac{1}{2}\left(\left\|e^{m+1}\right\|^{2}-\left\|e^{m}\right\|^{2}\right) .
$$

On the other hand, we have

$$
\begin{aligned}
& \left(\mathbf{U}^{m+1 / 2} \mathbf{U}_{x}^{m+1 / 2}-\mathbf{U}\left(t_{m+1 / 2}\right) \mathbf{U}_{x}\left(t_{m+1 / 2}\right), e^{m+1 / 2}\right) \\
& =\left(e^{m+1 / 2} e_{x}^{m+1 / 2}+\mathbf{U}\left(t_{m+1 / 2}\right) e_{x}^{m+1 / 2}+\mathbf{U}_{x}\left(t_{m+1 / 2}\right) e^{m+1 / 2}, e^{m+1 / 2}\right),
\end{aligned}
$$

and finally, using periodicity we can conclude that

$$
\left(e_{x x x}^{m+1 / 2}-e_{x x x x x}^{m+1 / 2}, e^{m+1 / 2}\right)=0 .
$$

Keeping all the above estimates in mind, we have the following:

$$
\begin{aligned}
\frac{1}{2}\left(\left\|e^{m+1}\right\|^{2}-\left\|e^{m}\right\|^{2}\right) & \leq C \Delta t\left\|\mathbf{U}_{x}\left(t_{m+1 / 2}\right)\right\|_{\infty}\left\|e^{m+1 / 2}\right\|^{2}+C \Delta t^{3}\left\|e^{m+1 / 2}\right\| \\
& \leq C \Delta t^{5}+C \Delta t\left\|e^{m+1 / 2}\right\|^{2} .
\end{aligned}
$$

Using a standard argument, we conclude that (5.3) holds.

We now turn into the proof of existence of a sequence $\left\{\mathbf{U}^{n}\right\}_{n=0}^{M}$ satisfying (5.1). For this we shall use the following variant of the well-known fixed point theorem of Brouwer [19].

Lemma 5.1. Let $H$ be a finite-dimensional Hilbert space with inner product $(., .)_{H}$, and norm $\|.\|_{H}$. Let the map $f: H \rightarrow H$ be continuous. Suppose there exists $\beta>0$ such that $(f(K), K)_{H}>0$ for all $K$ with $\|K\|=\beta$. Then there exists $K^{*} \in H$, $\left\|K^{*}\right\| \leq \beta$ such that $f\left(K^{*}\right)=0$.

The argument of existence of $\left\{\mathbf{U}^{n}\right\}_{n=0}^{M}$ proceeds in an inductive way. Assume that $\left\{\mathbf{U}^{j}\right\}_{j=0}^{n}$ exists.

For $K \in S_{N}$, define $f: S_{N} \rightarrow S_{N}$ by

$$
(f(K), \phi)=\left(K-2 \mathbf{U}^{m}, \phi\right)+\frac{\Delta t}{4}\left(K K_{x}, \phi\right)+\frac{\Delta t}{2}\left(K_{x x x}-K_{x x x x x}, \phi\right), \quad \forall \phi \in S_{N} .
$$

Such a map exists by the Riesz representation theorem; the fact that $f$ is continuous follows easily from inverse inequalities. Furthermore, by periodicity, letting $\phi=K$

$$
(f(K), K)=\left(K-2 \mathbf{U}^{m}, K\right) \geq\|K\|\left(\|K\|-2\left\|\mathbf{U}^{m}\right\|\right) \geq\|K\|\left(\|K\|-2\left\|\mathbf{U}^{0}\right\|\right),
$$

from (5.4). Letting $\beta>2\left\|\mathbf{U}^{0}\right\|$, we deduce the existence via Lemma 5.1 of a $K^{*} \in S_{N}$ such that $f\left(K^{*}\right)=0$. Now letting $\mathbf{U}^{m+1}=K^{*}-\mathbf{U}^{m}$, we get from (5.8) that

$$
\left(\mathbf{U}^{m+1}-\mathbf{U}^{m}, \phi\right)+\Delta t\left(\mathbf{U}^{m+1 / 2} \mathbf{U}_{x}^{m+1 / 2}+\mathbf{U}_{x x x}^{m+1 / 2}-\mathbf{U}_{x x x x x}^{m+1 / 2}, \phi\right)=0, \quad \forall \phi \in S_{N},
$$

proving the existence of $\mathbf{U}^{m+1}$. 
For uniqueness, suppose that $\mathbf{V}^{m+1} \in S_{N}$ satisfies

$$
\left(\mathbf{V}^{m+1}-\mathbf{V}^{m}, \phi\right)+\Delta t\left(\mathbf{V}^{m+1 / 2} \mathbf{V}_{x}^{m+1 / 2}+\mathbf{V}_{x x x}^{m+1 / 2}-\mathbf{V}_{x x x x x}^{m+1 / 2}, \phi\right)=0 .
$$

Then using $E^{m}=\mathbf{U}^{m}-\mathbf{V}^{m}$, from (5.1) and (5.9) we have for all $\phi \in S_{N}$,

$$
\begin{aligned}
\left(E^{m+1}\right. & \left.-E^{m}, \phi\right) \\
& =-\Delta t\left(\mathbf{V}^{m+1 / 2} E_{x}^{m+1 / 2}+\mathbf{U}_{x}^{m+1 / 2} E^{m+1 / 2}+E_{x x x}^{m+1 / 2}-E_{x x x x x}^{m+1 / 2}, \phi\right) .
\end{aligned}
$$

We claim that as long as $U^{m}$ exists, we have

$$
\begin{array}{ll}
\left\|\mathbf{U}^{m}\right\|_{\infty} \leq C & \text { for all } m \\
\left\|\mathbf{U}_{x}^{m}\right\|_{\infty} \leq C & \text { for all } m
\end{array}
$$

Infact, Theorem 5.1 and some Sobolev inequalities yields,

$$
\begin{aligned}
& \left\|U^{m}-u\left(t_{m}\right)\right\|_{\infty} \leq C N^{1 / 2}\left\|U^{m}-u\left(t_{m}\right)\right\| \leq C N^{1 / 2}\left(\frac{1}{N^{r}}+\Delta t^{2}\right) \\
& \left\|U_{x}^{m}-u_{x}\left(t_{m}\right)\right\|_{\infty} \leq C N^{1 / 2}\left\|\left(U^{m}-u\left(t_{m}\right)\right)_{x}\right\| \leq C N^{3 / 2}\left(\frac{1}{N^{r}}+\Delta t^{2}\right) .
\end{aligned}
$$

Using the facts $r \geq 2$, and (5.2), we deduce (5.10) and (5.11).

Letting $\phi=E^{m+1 / 2}$, we deduce that

$$
\begin{aligned}
\frac{1}{2}\left(\left\|E^{m+1}\right\|^{2}-\left\|E^{m}\right\|^{2}\right) \leq C \Delta t\left\|\mathbf{U}_{x}^{m+1 / 2}\right\|_{\infty} & \left\|E^{m+1 / 2}\right\|^{2} \\
& +C \Delta t\left\|\mathbf{V}_{x}^{m+1 / 2}\right\|_{\infty}\left\|E^{m+1 / 2}\right\|^{2},
\end{aligned}
$$

from the above relation, we conclude that

$$
\left\|E^{m+1}\right\|^{2} \leq \nu\left\|E^{m}\right\|^{2}, \quad \text { with } \quad \nu \leq 1 .
$$

Now taking $\mathbf{U}^{m}=\mathbf{V}^{m}$, we see that $E^{m+1}=0$, hence uniqueness follows.

Remark 5.1. In conclusion, combining the results of Theorems 3.1 and 5.1, we see that under the hypotheses of Theorem 5.1, the fully discrete scheme (5.1) satisfies the error estimate

$$
\max _{0 \leq m \leq M}\left\|u\left(t_{m}\right)-\mathbf{U}^{m}\right\| \leq C\left(\frac{1}{N^{r}}+\Delta t^{2}\right) .
$$

\section{REFERENCES}

[1] J. L. Bona, V. A. Dougalis, and O. A. Karakashian: Fully discrete Galerkin methods for the Korteweg-de Vries equation. IMA preprint series., 182, september 1985.

[2] G. A. Baker, V. A. Dougalis, and O. A. Karakashian: Convergence of Galerkin approximations for the Korteweg-de Vries equation. Mathematics of Computations., Vol 40, N-162, 419-433 (1983).

[3] Juan Carlos Ceballos., Mauricio Sepulveda., Octavio Paulo Vera Villagran.: The Korteweg-de Vries-Kawahara equation in a bounded domain and some numerical results. Applied Matheamatics and Computation., 190: 912-936, 2007.

[4] Shang Bin CUI., Dong Gao DENG., Shuang Ping TAO.: Global existence of solutions for the Cauchy problem of the Kawahara equation with $L^{2}$ initial data. Acta Mathematica Sinica., vol. 22 No. 5 : 1457-1466, 2006.

[5] M.T.Darvishi., F.Khani.: Numerical and explicit solutions of the fifth-order Korteweg-de Vries equations. Chaos, Solitons and Fractals., 39: 2484-2490, 2009.

[6] Gleb G. Doronin, Nikolai A. Larkin.: Well and ill-posed problems for the KdV and Kawahara equations. Bol. Soc. Paran. Mat., v. 26 1-2: 133-137, 2008. 
[7] Jennifer Gorsky, A. Alexandrou Himonas,: Well-posedness of KdV with higher dispersion. Mathematics and Computors in Simulation., 80 : 173-183, 2009.

[8] Hunter, J.K, Scheurle, J.: Existence of perturbed solitary wave solutions to a model equation for water waves. Physica D., 32, 253-268, 1988.

[9] T. Kato.: On the Korteweg-de Vries equation. Manuscripta Math., 28: 89-99, 1979.

[10] T. Kato.: On the Cauchy problem for the (generalized) Korteweg-de Vries equations. Stud. Appl. Math. Adv. Math. Suppl. Stud., 8: 93-128, 1983.

[11] T. Kawahara.: Oscillatory solitary waves in dispersive media. J. Phys. Soc. Japan., 33: 260264, 1972

[12] S. Kichenassamy, Olver, P. J.: Existence and nonexistence of solitary wave solutions to higher-order model evolution equations. SIAM J. Math. Anal., 23: 1141-1166, 1992.

[13] U. Koley: Convergence of numerical schemes for the Korteweg-de Vries-Kawahara equation. Submitted.

[14] Y. Maday, and A. Quarteroni: Error analysis for spectral approximation of Korteweg-de Vries equation. RAIRO- M2AN., 22, N-3 (1988), P. 499-529.

[15] B. Pelloni, and V. A. Dougalis: Error estimates for a fully discrete spectral scheme for a class of nonlinear, nonlocal dispersive wave equations. Applied Numerical Mathematics., 37 (2001), P. 95-107.

[16] J. Ponce.: Regularity of solutions to nonlinear dispersive equations. J. Diff. Eq., 78: 122-135, 1989.

[17] Mauricio Sepulveda., Octavio Paulo Vera Villagran.: Numerical method for a transport equation perturbed by dispersive terms of 3rd and 5th order. SCIENTIA. Series A: Mathematical Sciences., Vol 13: 13-21, 2006.

[18] A. Sjöberg.: On the Korteweg-de Vries equation: Existence and uniqueness. J. Math. Anal. Appl., 29: 569-579, 1970.

[19] R. Temam.: Navier-Stokes equations: Theory and Numerical Analysis. rev ed, NorthHolland., Amsterdam,1979.

[20] O. Vera.: Gain of regularity for a Korteweg-de Vries-Kawahara type equation. EJDE., 71: $1-24,2004$.

[21] Hua WANG., Shang Bin CUI., Dong Gao DENG.: Global existence of solutions for the Kawahara equation in Sobolev spaces of negative indices. Acta Mathematica Sinica., vol. 23 No. $8:$ 1435-1446, 2007.

(Ujjwal Koley)

Centre of Mathematics for Applications (CMA)

UNIVERSITY OF OSLO

P.O. BOX 1053, BLINDERN

$\mathrm{N}-0316$ OSLO, NoRWAY

E-mail address: toujjwal@gmail.com 